\title{
Análisis exploratorio sobre violencia filio-parental en una muestra de adolescentes mexicanos*
}

\author{
Exploratory analysis of child-parent violence \\ in a sample of Mexican adolescents
}

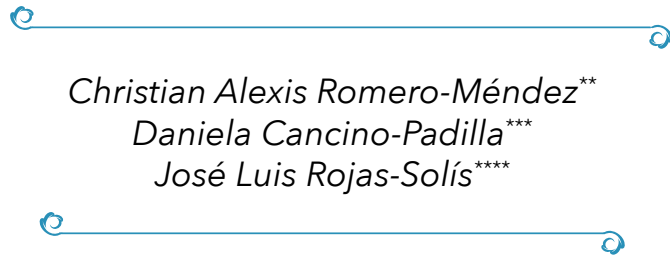

Recibido 27. 03. 2020 • Arbitrado 20. 04. 2020 •

Aprobado 25.05. 2020

* Estudio realizado entre agosto de 2017 y diciembre de 2020, dentro de la Facultad de Psicología de la Benemérita Universidad Autónoma de Puebla (México), como parte de la formación de jóvenes investigadores en estancias Delfín y AMC bajo la guía del Dr. José Luis Rojas-Solís.

* Egresado de la licenciatura en Psicología en la Universidad del Valle de Puebla (México) y becario del Programa XXIX Verano de la Investigación Científica 2019, Academia Mexicana de Ciencias. Correo electrónico: christianr.mendez97@gmail.com, ORCID: https:/ / orcid.org/0000-0003-48517116

*** Licenciada en Psicología por la Universidad Juárez Autónoma de Tabasco (México) y becaria del Programa XXII Verano de la Investigación Científica y Tecnológica del Pacífico, Programa Delfín 2017. Correo electrónico: danielacancino37@gmail.com, ORCID: https://orcid.org/00000001-8430-7218

- Doctor en Psicología por la Universidad de Salamanca (España) y Profesor-Investigador en la Benemérita Universidad Autónoma de Puebla (México). Correo electrónico: jlrojassolis@gmail.com, ORCID: http:// orcid.org/0000-0001-6339-4607

\section{Resumen}

El presente estudio tuvo como objetivo analizar la frecuencia de la violencia filio-parental (VFP) en una muestra de adolescente mexicanos, para ello se empleó un enfoque cuantitativo, con un diseño no experimental, de corte transversal $y$ ex post facto; con alcances exploratorios y descriptivos. La muestra fue seleccionada de manera no probabilística e intencional, participaron 407 adolescentes, 214 mujeres y 193 varones, con edades comprendidas entre los 12 a 18 años, los cuales fueron agrupados de acuerdo a la convivencia con sus padres o madres. El instrumento utilizado para detectar la VFP fue la Adaptación del Cuestionario de violencia filioparental en adolescentes mexicanos (Calvete y Veytia, 2018), el cual evalúa la frecuencia de la perpetración de la violencia psicológica y física de los hijos o hijas hacia sus progenitores. Los resultados señalaron la existencia de la violencia filio-parental, siendo la violencia psicológica cometida hacia la madre y hacia al padre la que con mayor frecuencia se ejerció por ambos sexos; 
datos que son congruentes con lo hallado en otras investigaciones. En las diferencias por sexo únicamente se identificó diferencia estadísticamente significativa para los adolescentes que indicaron vivir o haber vivido con ambos padres, siendo los varones mayormente perpetradores de violencia física hacia el papá en comparación con las mujeres. A manera de conclusión, se sugiere seguir explorando el fenómeno debido a que la literatura científica y académica continúa siendo escasa en regiones como México y América Latina.

Palabras clave: violencia filio parental, adolescente, mexicano

\section{Abstract}

The objective of this study was to analyze the frequency of child to parent violence (CPV) in a sample of Mexican adolescents. For this, a quantitative approach was used, with a non-experimental, cross-sectional and ex post facto design; with exploratory and descriptive scopes. The sample was selected in a non-probabilistic and intentional way, 407 adolescents, 214 women and 193 men, aged between 12 to 18 years, participated, which were grouped according to coexistence with their parents. The instrument used to detect CPV was the Adaptation of the Questionnaire on child-parent violence in Mexican adolescents (Calvete and Veytia, 2018), which assesses the frequency of the perpetration of psychological and physical violence by sons or daughters towards their parents. The results indicated the existence of filio-parental violence, being the psychological violence committed towards the mother and towards the father the one that was most frequently carried out by both sexes; data that is consistent with what was found in other investigations. In the differences by sex, a statistically significant difference was only identified for the adolescents who indicated living or having lived with both parents, the men being mostly perpetrators of physical violence towards the father compared to the women. In conclusion, it is suggested to continue exploring the phenomenon because the scientific and academic literature continues to be scarce in regions such as Mexico and Latin America.

Key word: Child to parent violence, adolescent, Mexican

\section{Introducción}

La familia es considerada como un subgrupo dentro de la sociedad donde la cualidad principal es el parentesco (Oliva y Villa, 2014), se trata de un grupo ampliamente estudiado debido a que su estructura e interacción pueden determinar el desarrollo de un individuo, pues en ese contexto se adquieren los aspectos básicos de socialización, como creencias, valores y actitudes (Amar, 
Abello y Tirado, 2004), que ayudan a la formación y establecimiento de relaciones interpersonales sanas (Mayor y Salazar, 2019). Pese a lo anterior, la familia no es ajena a la aparición de diversos fenómenos y problemáticas como la violencia intrafamiliar (Almenares, Louro y Ortiz, 1999), la cual se define como el uso sistematizado de actitudes y/o comportamientos de abuso que ejerce un integrante de la familia hacia otro, causando daños a su integridad física o psicológica (Mayor y Salazar, 2019).

Es así como en la literatura nacional e internacional se ha observado un gran auge en los estudios relacionados con esta problemática, pues se ha demostrado que su impacto no solamente recae en las graves consecuencias que genera sobre sus víctimas, sino que puede ser precursora de otros fenómenos indeseados. No obstante, las pesquisas realizadas hasta el momento suelen enfocarse en el estudio de la violencia doméstica o el maltrato infantil, omitiendo otras problemáticas como la violencia filio-parental (VFP) (Ibabe, 2019), la cual, pese a ser un objeto de estudio relativamente nuevo en contextos hispanoparlantes, tiene ya un gran desarrollo en países anglosajones y europeos (Calvete y Veyta, 2018; Loinaz, Andrés-Pueyo y Pereira, 2017; Pereira, 2017).

En este orden de ideas, la violencia filio-parental (VFP) es un fenómeno que se ha denominado de diferentes formas, por ejemplo, violencia ascendiente, maltrato filial, hijos maltratadores, o, síndrome de padres/progenitores maltratados (Aroca y Alba, 2012). Se trata de un fenómeno que surge como un subtipo de la violencia intrafamiliar (Agnew y Huguley, 1989; Calvete, Orue y Gámez-Guadix, 2012; Holt, 2016; Pereira et al., 2017), la cual se ha definido como el ejercicio de violencia física, verbal, psicológica y económica que ejercen los niños o adolescentes hacia sus progenitores o sustitutos de estos (Garrido y Galvis, 2016, Loinaz et al., 2017), con la finalidad principal de obtener poder, control y dominio sobre sus víctimas para conseguir lo que quieren (Aroca, Bellver, Alba, 2012). Es preciso indicar que en la definición de la VFP se recalca el uso intencionado de los actos violentos, realizados con conciencia, así como la constante reiteración de las conductas a lo largo del tiempo (Aroca y Alba, 2012). Por lo anterior, es esperable que la VFP esté captando la atención de la comunidad científica, aunque todavía es evidente su carácter exploratorio, especialmente, si se compara con investigaciones realizadas que abordan otras problemáticas que se presentan en la adolescencia como, por ejemplo, la violencia de pareja o el abuso sexual (Loinaz et al., 2017).

Por otra parte, los estudios realizados en diversos países han permitido identificar algunas características que presentan los hijos maltratadores y las familias donde la VFP está presente. En ese orden de ideas, se ha identificado que los agresores en su mayoría son adolescentes (Martínez, Estévez, Jiménez 
y Velilla, 2015) y el sexo predominante para cometer esta violencia es el masculino (Aroca-Montolío, Lorenzo-Moledo y Camilo Miró-Pérez, 2014). No obstante, la expresión de la violencia hacia los padres suele ser distinta, siendo los varones mayormente perpetradores de violencia física, mientras que las mujeres son mayormente perpetradoras de violencia psicológica (Ibabe y Jaureguizar, 2011). Así mismo, se ha identificado que los adolescentes agresores presentan algunas variables clínicas tales como la baja autoestima, sintomatología depresiva, impulsividad y baja regulación emocional, entre otras (Aroca-Montolío et al., 2014; Pereira, 2017). Por otro lado, Calvete y Veyta (2018) mencionan la relevancia de considerar a una agrupación diferente, como los adolescentes infractores o con antecedentes delictivos.

Ahora bien, entre las características de la familia donde existe la VFP, Patterson y MacCoby (1980) refieren que las madres son más propensas a recibir violencia en comparación a los padres, pues son ellas quienes tienen una mayor proximidad y convivencia con ellos. Del mismo modo, influye el hecho de que a las madres se les atribuiría el rol del sexo débil (Cottrell y Monk, 2004). Por otra parte, las familias monoparentales son más proclives para la presencia de la VFP (Aroca-Montolío et al., 2014), así como las familias donde la violencia intrafamiliar se ha presentado previamente (Contreras y Cano, 2016; Cottrel y Monk, 2004; Gallego, Novo, Fariña y Arce, 2019; Gámez-Guadix y Calvete, 2012), sugiriendo, incluso, un intercambio o bidireccionalidad de la violencia entre padres e hijos (Ibabe y Jaureguizar, 2011). Entre otros factores intervinientes se hallan los estilos de crianza, donde se muestra mayor frecuencia de la VFP en aquellos padres que son más permisivos, pues muestran dificultades para el establecimiento de límites y, por ende, el respeto (Pereira, 2017).

Históricamente, se puede ubicar literatura científica y académica sobre padres maltratados desde los años 50 y 80, sin embargo, ésta comenzó a tener mayor reconocimiento hasta hace apenas unos años (Aroca-Montolío et al., 2014). Al respecto es preciso señalar que la investigación de cada una de las vertientes de la violencia intrafamiliar implica diversos factores, tanto teóricos como metodológicos, que necesitan ser contemplados previo a la comprensión de un fenómeno (Poligero, 2016), es así como a lo largo de su estudio se han presentado dificultades que impiden su adecuada investigación. Dentro de la revisión de la literatura se pueden hallar dos principales razones.

En primer lugar, está la falta del establecimiento de una oportuna metodología para el adecuado estudio del fenómeno (March, 2017). En este aspecto se muestran los sesgos empleados en las investigaciones realizadas, como, por ejemplo, que el fenómeno de la VFP se analiza, solamente desde la perspectiva 
de los hijos agresores sin indagar como éste repercute, clínicamente en los padres (Rojas-Solís, Vázquez-Aramburu y Llamazares-Rojo, 2016). De igual manera existe una discrepancia en cuanto a la utilización de instrumentos que integren todos los tipos de violencia, debido a que existen algunos que solamente se enfocan en medir la violencia física o que algunos instrumentos solamente evalúan la violencia hacia uno de los padres (Calvete y Veytia, 2018; Calvete, Gámez-Guadix y Orue, 2014). Aunado a esto, se encuentran las dificultades en atribuir una definición operacional, donde se tiende a confundir los términos de "violencia hacia los padres" con "abuso/agresión hacia los padres" lo que trae consigo dificultades al momento de interpretar los resultados (Simmons, McEwan, Purcell y Ogloff, 2018).

En segundo lugar, en las fiscalías generales no se muestra un consenso exacto sobre los casos presentados debido a que los padres se muestran renuentes a reportar el abuso por parte de sus hijos por los estigmas sociales que puede desencadenar (Ibabe y Jaureguizar, 2011); tal vez por ello este tipo de violencia ha sido encubierta (Aroca-Montolío et al., 2014) e incluso negada por los padres (Webster, 2008), dificultando el acceso al conocimiento sobre su prevalencia (Condry y Miles, 2014; Contreras, Bustos-Navarrete y Cano-Lozano, 2019; Cottrel y Monk, 2004), así como su reconocimiento como una problemática actual y relevante.

En consonancia con lo anterior, es entendible que la VFP haya sido escasamente investigada en regiones como México y América Latina. En ese orden de ideas es preciso resaltar que la carencia de estudios no solamente dificulta la conceptualización del fenómeno de la VFP, sino que impide la implementación de programas para su prevención y tratamiento.

Por lo anteriormente expuesto, se expresa la necesidad de explorar el fenómeno de la VFP, por lo que el presente estudio pretende analizar su frecuencia en adolescentes hombres y mujeres y con ello identificar el tipo de violencia que más predomina, así como determinar a quién de ambos progenitores se dirigen con mayor frecuencia la violencia, $y$, por último, determinar las diferencias entre varones y mujeres en el ejercicio de la VFP.

En ese orden se plantearon las siguientes hipótesis de estudio: 1) el ejercicio de violencia psicológica tenga mayor frecuencia en comparación a la violencia física (Calvete, Orue y Sampedro, 2011); 2) la violencia psicológica será mayor hacia la madre (Contreras y Cano, 2016), mientras que 3) la violencia física será mayormente ejercida hacia el padre (Espinoza, Vivanco, Sepúlveda, Álvarez y Veliz, 2018) y, por último, 4) no se esperan diferencias estadísticamente significativas entre varones y mujeres quienes cometen violencia hacia sus padres (Gámez-Guadix y Calvete, 2012). 


\section{Método}

El presente estudio se realizó bajo un enfoque cuantitativo, utilizando un diseño no experimental de corte transversal, observacional y ex post facto con alcances exploratorios - descriptivos.

\section{Muestra}

La muestra fue seleccionada de manera no probabilística y por conveniencia, la cual estuvo constituida por 407 adolescentes estudiantes de secundaria y bachillerato del estado de Puebla (México) de los cuales 214 (52.6\%) fueron mujeres y 193 (47.4\%) varones, los criterios de inclusión establecidos para ser partícipes de la investigación fueron: 1) adolescentes con edades de entre los 12 a 18 años $(M=15.15$ y $D T=1.79), 2)$ que habiten o hayan habitado con mamá y/o papá y 3) ser alumnos activos en la institución educativa donde se obtuvo su participación.

La muestra de adolescentes se categorizó en tres grupos de acuerdo a la estructura familiar que indicaron: vivir o haber vivido con ambos padres, solamente con mamá y solamente con papá (ver Tabla 1).

Tabla 1. Agrupación de los adolescentes según su estructura familiar

\begin{tabular}{|c|c|c|c|c|c|c|c|c|}
\hline & \multirow{2}{*}{\multicolumn{2}{|c|}{ Sexo }} & \multicolumn{6}{|c|}{ Viven o han vivido con: } \\
\hline & & & \multicolumn{2}{|c|}{ Ambos padres } & \multicolumn{2}{|c|}{ Solo con mamá } & \multicolumn{2}{|c|}{ Solo con papá } \\
\hline & $\mathbf{F}$ & $\%$ & $\mathbf{F}$ & $\%$ & $\mathbf{F}$ & $\%$ & $\mathbf{F}$ & $\%$ \\
\hline Mujeres & 214 & 52.6 & 183 & 50.4 & 28 & 70 & 3 & 75 \\
\hline Varones & 193 & 47.4 & 180 & 49.6 & 12 & 30 & 1 & 25 \\
\hline
\end{tabular}

\section{Instrumentos}

En primera instancia se aplicó un cuestionario de datos sociodemográficos, el cual recogió información sobre el nombre de la institución educativa, edad, sexo, con quién viven o han vivido los o las participantes (ambos padres, solamente con mamá o solamente con papá).

Posteriormente se aplicó la Adaptación del cuestionario de violencia filio-parental en adolescentes mexicanos (Calvete y Veytia, 2018), el cual evalúa la frecuencia de violencia psicológica y física que ejercen los hijos hacia sus progenitores (mamá y papá), se conforma de 20 ítems, donde 10 corresponden a la VFP hacia la mamá y 10 a la VFP al papá. 7 de los 10 ítems se enfocan en la 
VFP psicológica, mientras que los 3 restantes en la VFP física. Los reactivos se evalúan a través de una escala Likert que va de 0 a 3 donde: $0=$ Nunca (no ha pasado en mi relación con mi madre o padre), $1=$ Rara vez (únicamente ha sucedido en 1 o 2 ocasiones), $2=$ Algunas veces (ha ocurrido entre 3 y 5 veces) y $3=$ Muy a menudo (se ha dado en 6 o más ocasiones). Los índices de consistencia interna obtenidos en la validación del instrumento en la población mexicana fueron de .89 para el factor de la violencia psicológica hacia la madre, .90 para la violencia psicológica hacia el padre, .93 para la violencia física contra la madre y .92 para la violencia física hacia el padre.

\section{Procedimiento}

En primera instancia se contactó con las autoridades de las instituciones educativas donde se les explicó la naturaleza y los objetivos de la investigación, una vez obtenido el permiso, se procedió a aplicar el cuestionario vía on-line a través de la plataforma de Google forms, para ello se requirió de las salas de cómputo de las instituciones educativas, el tiempo estimado para ser completado fue de 20 a 25 minutos, sin embargo, previo a que los adolescentes respondieran el cuestionario se les hizo conocedores de la garantía de confidencialidad y anonimato según lo establecido por la Sociedad Mexicana de Psicología (2010), de igual manera se recalcó la voluntariedad de su participación a través de un ítem ineludible que les otorgaba la opción de seguir con el cuestionario o en su defecto abandonarlo.

\section{Análisis estadísticos}

Se utilizó el sistema SPSS Statistical Package for the Social Sciences, versión 21, para Windows, donde en primer lugar se realizaron los análisis de fiabilidad y normalidad a través del índice de Alpha de Cronbach y el test de Kolgomorov Smirnov respectivamente; posteriormente se obtuvieron medidas de tendencia central y, como análisis inferenciales, se determinaron las diferencias por sexo entre varones y mujeres para dos tipos de convivencias (con ambos padres y solo con mamá) a través de la técnica de $U$ de Mann Whitney.

\section{Resultados}

En la Tabla 2 es posible observar que la distribución de los datos resultó no normal, mientras que la consistencia interna de las subescalas osciló entre el .65 y .95 lo que indica que su fiabilidad es buena. 
Tabla 2. Prueba de normalidad y análisis de fiabilidad en la muestra total y por sexo

\begin{tabular}{|c|c|c|c|c|c|c|}
\hline & & Subescalas & $K-S$ & $\begin{array}{c}\alpha \\
\text { Total }\end{array}$ & $\begin{array}{c}\alpha \\
\text { Mujeres }\end{array}$ & $\begin{array}{c}\alpha \\
\text { Hombres }\end{array}$ \\
\hline \multirow{4}{*}{ 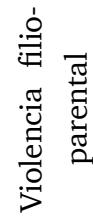 } & \multirow{2}{*}{ Mamá } & Psicológica & .000 & .74 & .73 & .75 \\
\hline & & Física & .000 & .68 & .72 & .65 \\
\hline & \multirow{2}{*}{ Papá } & Psicológica & .000 & .83 & .77 & .86 \\
\hline & & Física & .000 & .92 & .78 & .95 \\
\hline
\end{tabular}

Nota: $K-S=$ Kolmogorov Smirnov

En la Tabla 3 se encuentran las principales medidas de tendencia central, donde se puede observar que las conductas con mayor frecuencia se hallan en la VFP psicológica en los tres tipos se convivencia, no obstante, los resultados muestran que las madres tienden a ser mayormente receptoras de violencia psicológica, mientras que el padre es más receptor de violencia física.

Tabla 3. Principales medidas de tendencia central

\begin{tabular}{|c|c|c|c|c|c|c|c|c|}
\hline & & & & $\bar{x}$ & $M d$ & DT & Mín & Máx \\
\hline \multirow{12}{*}{ 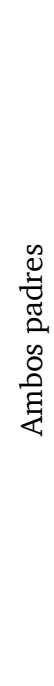 } & \multirow{6}{*}{ 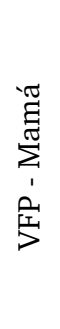 } & \multirow{3}{*}{ Psicológica } & Total & .50 & .42 & .4399 & .00 & 2.57 \\
\hline & & & Mujeres & .46 & .42 & .4068 & .00 & 2.57 \\
\hline & & & Hombres & .55 & .42 & .4682 & .00 & 2.57 \\
\hline & & \multirow{3}{*}{ Física } & Total & .07 & .00 & .2542 & .00 & 2.00 \\
\hline & & & Mujeres & .05 & .00 & .2102 & .00 & 1.67 \\
\hline & & & Hombres & .10 & .00 & .2907 & .00 & 2.00 \\
\hline & \multirow{6}{*}{ 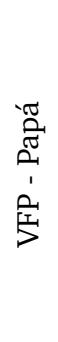 } & & Total & .41 & .28 & .5065 & .00 & 3.00 \\
\hline & & Psicológica & Mujeres & .37 & .28 & .4320 & .00 & 2.57 \\
\hline & & & Hombres & .44 & .28 & .5713 & .00 & 3.00 \\
\hline & & & Total & .11 & .00 & .4436 & .00 & 3.00 \\
\hline & & Física & Mujeres & .06 & .00 & .2740 & .00 & 2.33 \\
\hline & & & Hombres & .17 & .00 & .5608 & .00 & 3.00 \\
\hline
\end{tabular}




\begin{tabular}{|c|c|c|c|c|c|c|c|c|}
\hline & & & & $\bar{x}$ & $M d$ & DT & Mín & Máx \\
\hline \multirow{6}{*}{ 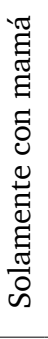 } & \multirow{6}{*}{ 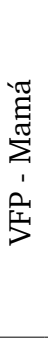 } & \multirow{3}{*}{ Psicológica } & Total & .55 & .57 & .4245 & .00 & 2.29 \\
\hline & & & Mujeres & .46 & .42 & .3226 & .00 & 1.14 \\
\hline & & & Hombres & .75 & .71 & .5685 & .00 & 2.29 \\
\hline & & \multirow{3}{*}{ Física } & Total & .02 & .00 & .1166 & .00 & .67 \\
\hline & & & Mujeres & .03 & .00 & .1387 & .00 & .67 \\
\hline & & & Hombres & .00 & .00 & .0000 & .00 & .00 \\
\hline \multirow{6}{*}{ 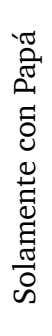 } & \multirow{6}{*}{ 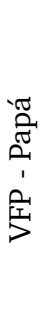 } & \multirow{4}{*}{ Psicológica } & Total & .46 & .35 & .2704 & .29 & .86 \\
\hline & & & Mujeres & .47 & .28 & .3299 & .29 & .86 \\
\hline & & & Hombres & .42 & .42 & .0000 & .43 & .43 \\
\hline & & & Total & .00 & .00 & .0000 & .00 & .00 \\
\hline & & \multirow[t]{2}{*}{ Física } & Mujeres & .00 & .00 & .0000 & .00 & .00 \\
\hline & & & Hombres & .00 & .00 & .0000 & .00 & .00 \\
\hline
\end{tabular}

Nota: $\overline{\mathrm{x}}=$ Media, $M d=$ Mediana, $D T=$ Desviación Típica, $M i n=$ Mínimo, Máx $=$ Máximo

Consecutivamente, en la Tabla 4 y 5, se muestra la distribución por ítem de la VFP psicológica y física, en los tres tipos de estructura familiar que los adolescentes indicaron habitar. En ese sentido, para los adolescentes que indicaron habitar con ambos padres el ítem con mayor frecuencia, para VFP psicológica a la mamá, fue el uno ("Le has gritado cuando estabas enojado/a"), tanto en mujeres como varones. Para el caso de la VFP psicológica al papá en la muestra de mujeres fue el ítem siete ("Has desobedecido en algo que te pidió y era importante para el/ella") y en la muestra de varones fue el ítem uno ("Le has gritado cuando estabas enojado/a").

En el caso de los adolescentes que solo habitan o habitaron con mamá el ítem con mayor frecuencia es el uno ("Le has gritado cuando estabas enojado/a"). Para el caso del tercer grupo los ítems con mayor frecuencia fueron el uno ("Le has gritado cuando estabas enojado/a"). y siete ("Has desobedecido en algo que te pidió y era importante para él/ella") en la muestra de mujeres, mientras que en los hombres fueron el uno ("Le has gritado cuando estabas enojado/a"), cuatro ("Le has chantajeado para conseguir lo que querías") y cinco ("Le has tomado dinero sin permiso").

El ítem con menor frecuencia, para todas las estructuras familiares, tanto en hombres como en mujeres fue el número dos ("Le has amenazado con pegarle, aunque no llegaste a hacerlo"). 
Tabla 4. Distribución de frecuencia por ítem en la violencia psicológica hacia los padres

\begin{tabular}{|c|c|c|c|c|c|c|c|c|c|}
\hline & & \multicolumn{4}{|c|}{ Mujeres } & \multicolumn{4}{|c|}{ Hombres } \\
\hline & \multirow[t]{2}{*}{$\stackrel{\Xi}{ \pm}$} & $\begin{array}{l}\text { J } \\
\mathbf{E} \\
\mathbf{Z}\end{array}$ & 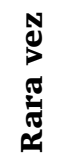 & $\begin{array}{c}\text { Algunas } \\
\text { veces }\end{array}$ & $\begin{array}{c}\text { Muy a } \\
\text { menudo }\end{array}$ & $\underset{\mathrm{Z}}{\mathrm{J}}$ & 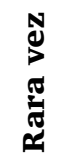 & $\begin{array}{c}\text { Algunas } \\
\text { veces }\end{array}$ & $\begin{array}{l}\text { Muy a } \\
\text { menudo }\end{array}$ \\
\hline & & $\mathbf{F}$ & $\mathbf{F}$ & $\mathbf{F}$ & $\mathbf{F}$ & $\mathbf{F}$ & $\mathbf{F}$ & $\mathbf{F}$ & $\mathbf{F}$ \\
\hline \multicolumn{10}{|c|}{ Con ambos padres } \\
\hline \multirow{6}{*}{ 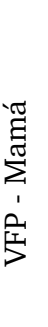 } & 1 & 58 & 75 & 40 & 10 & 48 & 75 & 40 & 10 \\
\hline & 2 & 172 & 9 & 2 & - & 163 & 13 & 3 & 1 \\
\hline & 3 & 148 & 28 & 6 & 1 & 126 & 42 & 9 & 3 \\
\hline & 4 & 142 & 33 & 7 & 1 & 124 & 47 & 8 & 1 \\
\hline & 5 & 141 & 37 & 3 & 2 & 135 & 33 & 9 & 3 \\
\hline & 6 & 95 & 58 & 27 & 3 & 87 & 65 & 19 & 9 \\
\hline \multirow{8}{*}{ 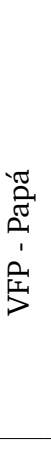 } & 7 & 84 & 73 & 17 & 9 & 83 & 59 & 26 & 12 \\
\hline & 1 & 101 & 51 & 27 & 4 & 96 & 51 & 22 & 11 \\
\hline & 2 & 174 & 2 & 7 & - & 160 & 13 & 3 & 4 \\
\hline & 3 & 151 & 21 & 8 & 3 & 129 & 33 & 9 & 9 \\
\hline & 4 & 136 & 36 & 9 & 2 & 131 & 30 & 14 & 5 \\
\hline & 5 & 154 & 21 & 6 & 2 & 144 & 26 & 5 & 5 \\
\hline & 6 & 122 & 41 & 16 & 4 & 117 & 43 & 12 & 8 \\
\hline & 7 & 97 & 61 & 18 & 7 & 103 & 49 & 20 & 8 \\
\hline \multicolumn{10}{|c|}{ Solo con mamá } \\
\hline \multirow{7}{*}{ 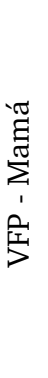 } & 1 & 9 & 8 & 9 & 2 & 2 & 5 & 2 & 3 \\
\hline & 2 & 27 & 1 & - & - & 11 & 1 & - & - \\
\hline & 3 & 23 & 3 & 1 & 1 & 8 & 3 & 1 & - \\
\hline & 4 & 25 & 3 & - & - & 7 & 3 & 1 & 1 \\
\hline & 5 & 23 & 4 & 1 & - & 9 & 2 & 1 & - \\
\hline & 6 & 10 & 16 & 1 & 1 & 3 & 4 & 4 & 1 \\
\hline & 7 & 12 & 12 & 3 & 1 & 4 & 5 & 2 & 1 \\
\hline
\end{tabular}




\begin{tabular}{|c|c|c|c|c|c|c|c|c|c|}
\hline & & & & Mujeres & & & & lombres & \\
\hline & $\stackrel{\Xi}{ \pm}$ & 导 & 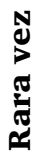 & $\begin{array}{c}\text { Algunas } \\
\text { veces }\end{array}$ & $\begin{array}{c}\text { Muy a } \\
\text { menudo }\end{array}$ & $\begin{array}{l}\text { 巳 } \\
\text { Z } \\
\text { Z }\end{array}$ & 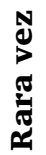 & $\begin{array}{c}\text { Algunas } \\
\text { veces }\end{array}$ & $\begin{array}{c}\text { Muy a } \\
\text { menudo }\end{array}$ \\
\hline & & $\mathbf{F}$ & $\mathbf{F}$ & $\mathbf{F}$ & $\mathbf{F}$ & $\mathbf{F}$ & $\mathbf{F}$ & $\mathbf{F}$ & $\mathbf{F}$ \\
\hline & & & & & Solo con & & & & \\
\hline & 1 & - & 2 & 1 & - & - & 1 & - & - \\
\hline & 2 & 3 & - & - & - & 1 & - & - & - \\
\hline 范 & 3 & 2 & 1 & - & - & 1 & - & - & - \\
\hline ه & 4 & 3 & - & - & - & - & 1 & - & - \\
\hline 5 & 5 & 2 & 1 & - & - & - & 1 & - & - \\
\hline & 6 & 3 & - & - & - & 1 & - & - & - \\
\hline & 7 & - & 2 & 1 & - & 1 & - & - & - \\
\hline
\end{tabular}

Nota: 1 = Le has gritado cuando estabas enojado/a, $2=$ Le has amenazado con pegarle, aunque no llegaste a hacerlo, 3 = Le has insultado o dicho groserías, $4=$ Le has chantajeado para conseguir lo que querías, $5=$ Le has tomado dinero sin permiso, $6=$ Has hecho algo para molestarle, $7=$ Has desobedecido en algo que te pidió y era importante para el/ella.

Para el caso de la VFP física, es posible observar, los adolescentes que indicaron vivir o haber vivido con ambos padres, tanto varones y mujeres, el ítem con mayor frecuencia fue el uno ("Le has empujado o golpeado en una pelea") y el que tuvo menor frecuencia fue el dos ("Le has golpeado con algo que podía hacer daño").

En el caso de las mujeres que solo indicaron vivir o haber vivido con mamá el ítem con mayor frecuencia fue el dos ("Le has golpeado con algo que podía hacer daño"), mientras que los varones indicaron nunca haber ejercido VFP física. Por otro lado, es preciso señalar que los adolescentes quienes viven solamente con papá indicaron nunca haber ejercido violencia VFP hacia ellos.

Tabla 5. Distribución de frecuencia por item en la violencia fisica hacia los padres

\begin{tabular}{|c|c|c|c|c|c|c|c|c|c|}
\hline & & \multicolumn{4}{|c|}{ Mujeres } & \multicolumn{4}{|c|}{ Hombres } \\
\hline & $\stackrel{\Xi}{ \pm}$ & 导 & 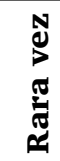 & $\begin{array}{c}\text { Algunas } \\
\text { veces }\end{array}$ & $\begin{array}{c}\text { Muy a } \\
\text { menudo }\end{array}$ & $\begin{array}{l}\text { 巳ु } \\
\text { Z } \\
\mathbf{Z}\end{array}$ & 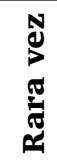 & $\begin{array}{c}\text { Algunas } \\
\text { veces }\end{array}$ & $\begin{array}{l}\text { Muy a } \\
\text { menudo }\end{array}$ \\
\hline & & $\mathbf{F}$ & $\mathbf{F}$ & $\mathbf{F}$ & $\mathbf{F}$ & $\mathbf{F}$ & $\mathbf{F}$ & $\mathbf{F}$ & $\mathbf{F}$ \\
\hline \multicolumn{10}{|c|}{ Con ambos padres } \\
\hline \multirow{3}{*}{ 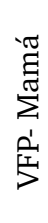 } & 1 & 170 & 13 & - & - & 158 & 18 & 4 & - \\
\hline & 2 & 177 & 4 & 2 & - & & 9 & - & 1 \\
\hline & 3 & 178 & 2 & 3 & - & & 9 & 1 & 2 \\
\hline
\end{tabular}




\begin{tabular}{|c|c|c|c|c|c|c|c|c|c|}
\hline & & \multicolumn{4}{|c|}{ Mujeres } & \multicolumn{4}{|c|}{ Hombres } \\
\hline & $\stackrel{\Xi}{\Xi}$ & 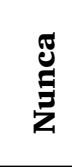 & 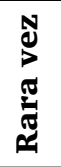 & $\begin{array}{c}\text { Algunas } \\
\text { veces }\end{array}$ & $\begin{array}{c}\text { Muy a } \\
\text { menudo }\end{array}$ & 导 & 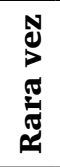 & $\begin{array}{c}\text { Algunas } \\
\text { veces }\end{array}$ & $\begin{array}{c}\text { Muy a } \\
\text { menudo }\end{array}$ \\
\hline & & $\mathbf{F}$ & $\mathbf{F}$ & $\mathbf{F}$ & $\mathbf{F}$ & $\mathbf{F}$ & $\mathbf{F}$ & $\mathbf{F}$ & $\mathbf{F}$ \\
\hline \multirow{3}{*}{ 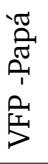 } & 1 & 172 & 7 & 4 & - & 158 & 12 & 6 & 4 \\
\hline & 2 & 179 & 1 & 2 & 1 & & 5 & 5 & 4 \\
\hline & 3 & 177 & 3 & 2 & 1 & & 9 & 6 & 4 \\
\hline \multicolumn{10}{|c|}{ Solo con mamá } \\
\hline \multirow{3}{*}{ 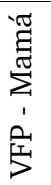 } & 1 & 26 & 2 & - & - & 12 & - & - & - \\
\hline & 2 & 28 & - & - & - & & - & - & - \\
\hline & 3 & 27 & 1 & - & - & & - & - & - \\
\hline
\end{tabular}

Solo con papá

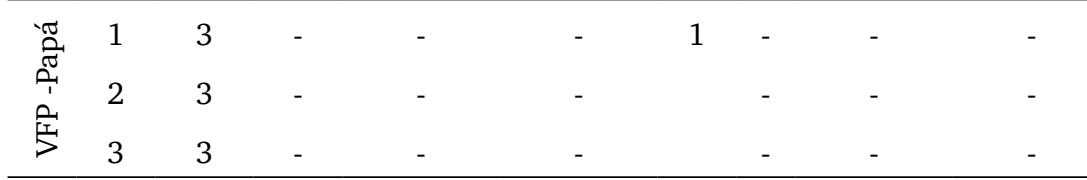

Nota: 1 = Le has empujado o golpeado en una pelea, 2 = Le has golpeado con algo que podía hacer daño, 3 = Le diste una patada o puñetazo.

\section{Diferencias por sexo}

Se realizaron las diferencias por sexo en los adolescentes varones y mujeres que habitaban o habían habitado con ambos padres y solamente con mamá. En el caso de las y los adolescentes que solamente viven o habían vivido con su papá no se realizaron los análisis inferenciales debido al escaso número de participantes. Los análisis se realizaron a través de la técnica de $U$ de Mann Whitney y los valores se consideraron significativos si el resultado de $p$ era igual o menor a punto cero cinco.

Así, en la muestra de adolescentes que habitan con ambos padres no se hallaron diferencias estadísticamente significativas para la violencia psicológica a la mamá entre mujeres $(M d=.42, n=183$ ) y hombres ( $M d=.42$, $\mathrm{n}=180), U=14801.000, z=-1.684, p=.092, r=-.088$, Psest $=.44$. Esto mismo fue encontrado en las variables de la violencia física a la mamá cometida por mujeres $(M d=.00, n=183)$ y hombres $(M d=.00, \mathrm{n}=180), U=15480.000$, $z=-1.748, p=.080, r=-.091$, Psest $=.46$, y en la violencia psicológica al papá, de igual manera, no se hallaron diferencias entre mujeres $(M d=.28$, $\mathrm{n}=183)$ y hombres $(M d=.28, \mathrm{n}=180), U=15763.000, \mathrm{z}=-.718, p=.473$, $r=-.037$, Psest $=.47$. No obstante para la violencia física al papá sí se hallaron 
diferencias estadísticamente significativas entre mujeres $(M d=.00, \mathrm{n}=183)$ y hombres ( $M d=.00, n=180), U=15385.500, z=-2.019, p=.044, r=-.105$, Psest $=.46$, siendo mayormente cometida por varones.

Para el caso de adolescentes que solamente habitan con mamá no se hallaron diferencias estadísticamente significativas en la violencia psicológica hacia la mamá cometida por mujeres $(M d=.46, \mathrm{n}=28)$ y hombres $(M d=.75$, $\mathrm{n}=12), U=114.000, z=-1.609, p=.108, r=-.254$, Psest $=.28$, los mismos hallazgos fueron encontrados en la violencia física a la mamá cometida por mujeres $(M d=.00, n=28)$ y hombres $(M d=.00, \mathrm{n}=12), U=156.000, z=-.938$, $p=.348, r=-.148$, Psest $=.39$.

\section{Discusión}

El presente estudio tuvo como objetivo analizar la frecuencia de la VFP, así como determinar las diferencias por sexo en hombres y mujeres. En ese orden, los resultados obtenidos permiten aceptar la primera hipótesis planteada, donde se esperaba que la violencia psicológica sería la que tendría mayor frecuencia, como pudo observarse en los tres tipos de estructura familiar que indicaron los adolescentes, la violencia psicológica tuvo mayor frecuencia en comparación a la física, consistente a lo que se ha encontrado dentro de la evidencia empírica acumulada (Simmons et al., 2018). Al respecto se han mencionado las dificultades que se han identificado para tener un consenso sobre la VFP psicológica, debido a que los estudios evalúan este tipo de violencia con otras definiciones operacionales, tales como "violencia verbal" o "violencia emocional". Aunado a esto, en torno a las explicaciones que se le han atribuido a su mayor frecuencia sobre los otros tipos de violencia, es debido a que la violencia psicológica tiene mayor facilidad de pasar desapercibida y ser perpetrada, dado que las consecuencias que genera no son visibles como las que causan la violencia física o sexual, en ese sentido, su ejercicio puede ser considerado, incluso, como algo normal en la vida cotidiana (Hirigoyen, 2005).

Por otra parte, pese a que se esperaba que las madres serían mayormente víctimas de VFP, porque podrían ser socialmente percibidas más débiles que los padres (Ibabe y Jaureguizar, 2011), los datos del presente estudio señalaron que ambos padres son receptores de violencia, aunque de manera diferente, siendo las madres mayormente receptoras de violencia psicológica (Contreras y Cano, 2016). En ese sentido, en la distribución por ítem, se pudo hallar que los y las adolescentes ejercieron con mayor frecuencia conductas de violencia psicológica hacia la madre, tales como haberle gritado cuando 
se encontraban enojados, lo que constituye una aproximación a lo señalado por Calvete et al. (2011), quienes refieren que las agresiones de tipo verbal son mayores hacia las madres. Por otro lado, se mostró que la violencia física suele ser mayor hacia los padres, acorde a lo expuesto por Browne y Hamilton (1998), quienes señalan que los padres son más propensos a sufrir conductas severas, las cuales pueden ir desde golpes e incluso amenazas con un objeto o arma. Los resultados anteriores permitieron aceptar la segunda y tercera hipótesis respectivamente.

Ahora bien, en la cuarta hipótesis se planteó que no existirían diferencias estadísticamente significativas entre varones y mujeres para la perpetración de la violencia hacia sus padres en ninguna de sus modalidades, esto acorde a lo que se ha encontrado dentro de la revisión sistemática realizada por Simmons et al. (2018), quienes en una compilación y análisis de estudios publicados en los últimos 60 años sobre la violencia filio-parental hallaron que, en muestras representativas comunitarias, no se había encontrado diferencias significativas para la perpetración de la violencia física. No obstante, esta hipótesis fue rechazada debido a que en el presente estudio sí se hallaron diferencias por sexo específicamente en adolescentes quienes habitan con ambos padres, mostrando que la violencia física hacia el papá es más frecuentemente cometida por varones en comparación con las mujeres, hallazgos que son consistentes con otros estudios (Aroca-Montlío et al., 2014; Boxer, Gullan y Mahoney, 2009; Espinoza et al., 2018). Aunado a esto, se ha mencionado que los adolescentes varones muestran una mayor aceptación del ejercicio de estas conductas hacia los padres, hecho que desencadena vergüenza y culpa cuando los mismos actos violentos en naturaleza física son ejercidos hacia la madre (Ibabe y Jaureguizar, 2011).

Entre otros hallazgos importantes, es preciso mencionar que las familias monoparentales suelen estar conformadas solo por la mamá e hijos o hijas (Pereira, 2017), tal como pudo observarse en el presente estudio. Con relación a lo anterior se encontró que la violencia psicológica en las familias monoparentales, específicamente, en las que se convive con la mamá es más frecuente en comparación con los y las adolescentes que habitan con ambos padres. Congruente a lo señalado por Aroca-Montolío et al. (2014), este hecho puede explicarse por diversos factores tales como: 1) que las madres son percibidas más débiles, 2) son más fáciles de manipular debido a la responsabilidad de la que son cargo, generado sentimientos de culpa ante alguna conducta disruptiva del hijo, 3) experiencias de maltrato previas por parte de hombres y 4) prejuicios sociales que le otorgan el rol subordinado (Pereira, 2017). 


\section{Conclusiones}

A manera de conclusión, el presente estudio muestra la existencia de la VFP en adolescentes mexicanos, datos similares a otros estudios llevados con población mexicana, donde se muestra su existencia (Calvete y Veytia, 2018; Vázquez-Sánchez, Romo-Tobón, Rojas-Solís, González y Yedra, 2019). Añadido a ello, se evidenció que ambos padres tienden a ser víctimas de violencia, aunque en distintas modalidades, siendo la madre mayormente víctima de violencia psicológica, mientras que el padre es mayormente víctima de violencia física. En la misma línea, se mostró que los adolescentes varones, quienes convivían con ambos padres, ejercieron con mayor frecuencia violencia física hacía el papá en comparación a las mujeres. Con base en los resultados obtenidos con anterioridad se recalca la necesidad de seguir explorando el fenómeno de la VFP, sobre todo, en regiones como México y América Latina, donde los antecedentes sobre investigaciones realizadas aún son escasos.

\section{Limitaciones y futuras líneas de investigación}

Finalmente es pertinente señalar que el presente estudio contó con algunas limitaciones tales como: la selección de la muestra la cual fue de manera no probabilística, dando como resultado que las estructuras familiares en las que se agruparon los adolescentes no hayan obtenido un número equivalente en participantes, aspecto que impide la generalización de los resultados. Futuras investigaciones podrían incluir una selección aleatoria y probabilística de la muestra, también sería pertinente analizar las dinámicas de la VFP desde la perspectiva de los padres, así como incluir otras variables con las que el fenómeno de la VFP se encuentra asociada.

\section{Agradecimientos}

La alumna Daniela Cancino-Padilla realizó parte de este artículo dentro del Verano de la Investigación Científica y Tecnológica del Pacífico, Programa Delfín; el alumno Christian Alexis Romero-Méndez realizó parte de este manuscrito dentro del Verano de la Investigación Científica - Academia Mexicana de Ciencias, Estudio realizado dentro del Cuerpo Académico (BUAP-CA-330): "Prevención de la violencia: Educando para una Cultura de Paz a través de la Participación Social". 


\section{Referencias}

Almenares, M., Louro, I., y Ortiz, M. T. (1999). Comportamiento de la violencia intrafamiliar. Revista Cubana de Medicina General Integral, 15, 285-292. Recuperado de: http://scielo.sld.cu/pdf/mgi/v15n3/mgi11399.pdf

Amar, J., Abello, R. y Tirado, D. (2004). Desarrollo infantil y construcción del mundo social. Colombia: Ediciones Uninorte.

Aroca, C. y Alba, J.L. (2012). La violencia filio-parental en hijos e hijas adolescentes con rasgos de psicopatía. Criminología y Justicia, 3, 25-44. Recuperado de: https:// dialnet.unirioja.es/descarga/articulo/4045978.pdf

Aroca, C., Bellver, M. C. y Alba, J. L. (2012). La teoría del aprendizaje social como modelo explicativo de la violencia filio-parental. Revista complutense de educación, 23(2), 487-511, doi:10.5209/rev_RCED.2012.v23.n2.40039

Aroca-Montolío, C., Lorenzo-Moledo, M. y Miró-Pérez, C. (2014). La violencia filio-parental: un análisis de sus claves. Anales de psicología, 30(1), 157-170. Recuperado de: http://www.redalyc.org/articulo.oa?id=16729452017

Agnew, R. y Huguley, S. (1989). Adolescent violence toward parents. Journal of marriage and family, 51(3), 699-711. Recuperado de: https://www.jstor.org/ stable/352169

Browne, K. D. y Hamilton, C. E. (1998). Physical violence between young adults and their parents: Associations with a history of child maltreatment. Journal of Family Violence, 13(1), 59-79. doi: 10.1023/A:1022812816957

Boxer, P., Gullan, R. L. y Mahoney, A. (2009). Adolescents' physical aggression toward parents in a clinic-referred sample. Journal of Clinical Child and Adolescent Psychology, 38(1), 106-116. doi:10.1080/15374410802575396

Calvete, E., Gámez-Guadix, M. y Orue, I. (2014). Características familiares asociadas a violencia filio-parental en adolescentes. Anales en psicología, 30(3), 1176-1182. doi: 10.6018/analesps.30.3.166291

Calvete, E., Orue, I. y Gámez-Guadix, M. (2012). Child-to-Parent Violence: Emotional and Behavioral Predictors. Journal of Interpersonal Violence, 28(4) 755 -772. doi: 10.1177/0886260512455869

Calvete, E., Orue, I. y Sampedro, R. (2011). Violencia filio-parental en la adolescencia: características ambientales y personales. Infancia y aprendizaje, 34(3), 349-363. doi: 10.1174/021037011797238577 
Calvete, E. y Veytia, M. (2018). Adaptación del cuestionario de violencia filio-parental en adolescentes mexicanos. Revista Latinoamericana de Psicología, 50(1), 49-60. doi: 10.14349/rlp.2018.v50.n1.5

Contreras, L. y Cano, M. (2016). Child-to-parent violence: The role of exposure to violence and its relationship to social-cognitive processing. The European Journal of Psychology Applied to Legal Context, 8, 43-50. doi: 10.1016/j.ejpal.2016.03.003

Contreras, L., Bustos-Navarrete, C. y Cano-Lozano, M. C. (2019). Child-to-parent Violence Questionnaire (CPV-Q): Validation among Spanish adolescents. International Journal of Clinical and Health Psychology, 19, 67-74. doi: 10.1016/j. ijchp.2018.09.001

Condry, R. y Miles, C. (2014). Adolescent to parent violence: Framing and mapping a hidden problema. Criminology \&amp; Criminal Justice, 14(3), 257-275. doi: $10.1177 / 1748895813500155$

Cottrel, B. y Monk, P. (2004). Adolescent-to-Parent Abuse A Qualitative Overview of Common Themes. Journal of family issues, 25(8), 1072-1095. doi: 10.1177/0192513X03261330

Espinoza, S. M., Vivanco, R. A., Sepúlveda, R. E., Álvarez, A. J. y Velíz, A. (2018). Violencia ejercida hacia ambos padres desde adolescentes que cursan la educación secundaria en la ciudad de Osorno, Chile. Espacios, 39(17), 33. http://revistapai. ucm.cl/article/download/156/151

Gallego, R., Novo, M., Fariña, F. y Arce, R. (2019). Child-to-parent Violence and Parent-to-child Violence: A Meta-analytic Review. The European Journal of Psychology Applied to Legal Context, 11(2), 51-59. doi: 10.5093/ejpalc2019a4

Gámez-Guadix, M. y Calvete, E. (2012). Child to parent violence and its association to parental violence exposition and parent to children aggression. Psicothema, 24, 277-283. Recuperado de: http://www.psicothema.com/pdf/4011.pdf

Garrido,V.yGalvisM.J.(2016).Laviolenciafilio-parental:unarevisióndelainvestigación empírica en España y sus implicaciones para la prevención y tratamiento. Revista de Derecho penal y Criminología, 3(16), 339-374. Recuperado de: http://e-spacio. uned.es/fez/view/bibliuned:revistaDerechoPenalyCriminologia-2016-16-5035

Hirigoyen, M. (2005). Mujeres maltratadas. Los mecanismos de la violencia en la pareja. Madrid: Paidós.

Holt, A. (2016). Adolescent-to-Parent abuse as a form of "domestic violence": A Conceptual Review. Trauma, violence, y abuse, 17(5) 490-499. doi: 10.1177/1524838015584372 
Ibabe, I. (2019). Adolescent-to-Parent Violence and Family Environment: The Perceptions of Same Reality? International journal of environmental research and public health, 16(2215), 2-14. doi: 10.3390/ijerph16122215

Ibabe, I. y Jaureguizar, J. (2011). ¿Hasta qué punto la violencia filio-parental es bidireccional? Anales de Psicología, 27(2), 265-277. Recuperado de: https://www. redalyc.org/articulo.oa?id $=16720051001$

Loinaz, I., Andrés-Pueyo, A. y Pereira, R. (2017). Factores de riesgo de violencia filio-parental: una aproximación con juicio de expertos. Acción Psicológica, 14(2), 17-32. doi: 10.5944/ap.14.2.20747

March, R. (2017). ¿Es siempre la familia el principal factor de riesgo en la violencia filioparental? Revista sobre la infancia y la adolescencia, 12, 1-29. doi: 10.4995/ reinad.2017.6433

Martínez, M. L., Estévez, E., Jiménez, T. I. y Velilla, C. (2015). Violencia filio-parental: Principales características, factores de riesgo y claves para la intervención. Childparent violence: Main characteristics, risk factors and keys to intervention. Papeles del Psicólogo, 36(3), 216-223. Recuperado de: http://www.papelesdelpsicologo. es/pdf/2615.pdf

Mayor, S. y Salazar, C. A. (2019). La violencia intrafamiliar. Un problema de salud actual. Gaceta médica espirituana, 21(1), 96-105. Recuperado de: http://scielo. sld.cu/pdf/gme/v21n1/1608-8921-gme-21-01-96.pdf

Oliva, E. y Villa, V., J. (2014). Hacia un concepto interdisciplinarios de la familia en la globalización. Justicia juris. 10(1), 11-20. Recuperado de: http://www.scielo. org.co/pdf/jusju/v10n1/v10n1a02.pdf

Patterson, G. R. y MacCoby, E. E. (1980). Mothers: The unacknowledged victims. Monographs of the society for research in child development,45(5), 1-64. doi: $10.2307 / 1165841$

Pereira, R. (2017). Violencia filio-parental: factores que favorecen su aparición. Revista Construção Psicopedagógica, 25(26) 5-16. Recuperado de: http://pepsic. bvsalud.org/pdf/cp/v25n26/02.pdf

Pereira R., Loinaz, I., Hoyo-Bilbao, J., Arrospide, J., Bertino L., Calvo, A., Montes, Y. y Gutierrez, M. (2017). proposal for a definition of filio-parental violence: consensus of the spanish society for the study of filio-parental violence (sevifip). Papeles del Psicólogo, 38(3), 216-223, doi: 10.23923/pap.psicol2017.2839

Poligero, A. M. (2016). La violencia filioparental en el contexto de la violencia familiar. IPSE-ds, 9, 69-84. Recuperado de: https://dialnet.unirioja.es/descarga/ articulo/6361584.pdf 
Rojas-Solís, J.L., Vázquez-Aramburu, G. y Llamazares-Rojo, J.A. (2016). Violencia filio-parental: una revisión de un fenómeno emergente en la investigación psicológica. Ajayu,14 (6), 140-161. Recuperado de: http://www.redalyc.org/ articulo.oa? $\mathrm{id}=461545454007$

Simmons, M., McEwan, T. E., Purcell, R. y Ogloff, J. (2018). Sixty years of child-toparent abuse research: What we know and where to go. Aggression and Violent Behavior, 38, 31-52. doi: 10.1016/j.avb.2017.11.001

Sociedad Mexicana de Psicología. (2010). Código ético del psicólogo. México, D.F.: Trillas.

Vázquez-Sánchez, V., Romo-Tobón, R. J., Rojas-Solís, J. L., González, M.,y Yedra, L. R. (2019). Violencia filio-parental en adultos emergentes mexicanos: un análisis exploratorio. Revista electrónica de psicología Iztacala, 22(3), 2534-2551. Recuperado de: https://www.medigraphic.com/pdfs/epsicologia/epi-2019/ epi193d.pdf

Webster, A. (2008). Adolescent to parent abuse: an overview. CDF Reader, 7(1), 4-8. doi: 10.1002/car.1053 